\title{
A novel analytical model of $M G M T$ methylation pyrosequencing offers improved predictive performance in patients with gliomas
}

\author{
Rui-Chao Chai ${ }^{1,2} \cdot$ Yu-Qing Liu, ${ }^{1,2} \cdot$ Ke-Nan Zhang ${ }^{1,2,3} \cdot$ Fan Wu ${ }^{1,2} \cdot$ Zheng Zhao ${ }^{1,2} \cdot$ Kuan-Yu Wang ${ }^{1,2,3} \cdot$ \\ Tao Jiang ${ }^{1,2,3} \cdot$ Yong-Zhi Wang ${ }^{1,2,3}$
}

Received: 9 July 2018 / Revised: 29 August 2018 / Accepted: 31 August 2018 / Published online: 5 October 2018

(c) United States \& Canadian Academy of Pathology 2018

\begin{abstract}
The methylation status of the promoter of $M G M T$ gene is a crucial factor influencing clinical decision-making in patients with gliomas. MGMT pyrosequencing results are often dichotomized by a cut-off value based on an average of several tested CpGs. However, this method frequently results in a "gray zone", representing a dilemma for physicians. We therefore propose a novel analytical model for MGMT methylation pyrosequencing. MGMT $\mathrm{CpG}$ heterogeneity was investigated in 213 glioma patients in two tested cohorts: cohort A in which CpGs 75-82 were tested and cohort B in which CpGs 72-78 were tested. The predictive performances of the novel and traditional averaging models were compared in 135 patients who received temozolomide using receiver operating characteristic curves and Kaplan-Meier curves, and in patients stratified according to isocitrate dehydrogenase gene mutation status. The results were validated in an independent cohort of 65 consecutive patients with high-grade gliomas from the Chinese Glioma Genome Atlas database. Heterogeneity of MGMT promoter $\mathrm{CpG}$ methylation level was observed in most gliomas. The optimal cut-off value for each individual $\mathrm{CpG}$ varied from 4-16\%. The current analysis defined MGMT promoter methylation as occurring when at least three CpGs exceeded their respective cut-off values. This novel analysis could accurately predict the prognosis of patients in the methylation "gray zone" according to the standard averaging method, and improved the area under the curves from $0.67,0.76$, and 0.67 to 0.70 , 0.84 , and 0.72 in cohorts $\mathrm{A}, \mathrm{B}$, and the validation cohort, respectively, demonstrating superiority of this analytical method in all three cohorts. Furthermore, the advantages of the novel analysis were retained regardless of WHO grade and isocitrate dehydrogenase gene mutation status. In conclusion, this novel analytical model offers an improved clinical predictive performance for $M G M T$ pyrosequencing results and is suitable for clinical use in patients with gliomas.
\end{abstract}

These authors contributed equally: Rui-Chao Chai, Yu-Qing Liu, Ke-Nan Zhang.

Electronic supplementary material The online version of this article (https://doi.org/10.1038/s41379-018-0143-2) contains supplementary material, which is available to authorized users.

Yong-Zhi Wang

yongzhiwang_bni@163.com

1 Department of Molecular Neuropathology, Beijing Neurosurgical Institute, Capital Medical University, Beijing, China

2 Department of Neurosurgery, Beijing Tiantan Hospital, Capital Medical University, Beijing, China

3 Chinese Glioma Genome Atlas, Beijing, China

\section{Introduction}

$\mathrm{O}_{6}$-methylguanine-DNA methyltransferase $(M G M T)$ promoter methylation is a useful predictive biomarker in relation to temozolomide chemotherapy, as demonstrated in a series of studies and clinical trials [1-5]. MGMT methylation testing has been established as a routine molecular pathological technique for patients with glioma. Pyrosequencing is a robust technique for quantifying the MGMT $\mathrm{CpG}$ methylation level compared with methylation-specific PCR, which has been used in most clinical trials [2, 6-10]. Pyrosequencing has been regarded as the "gold standard" for MGMT promoter methylation testing [9, 11-17], and is recommended by clinical glioma guidelines [18].

The MGMT promoter contains 98 individual CpGs surrounding the transcription start site (Fig. 1). The methylation statuses of CpGs 72-90, localized in exon 1 and subsequent intron 1 , have been shown to have a major 
A

CpG island in MGMT promoter
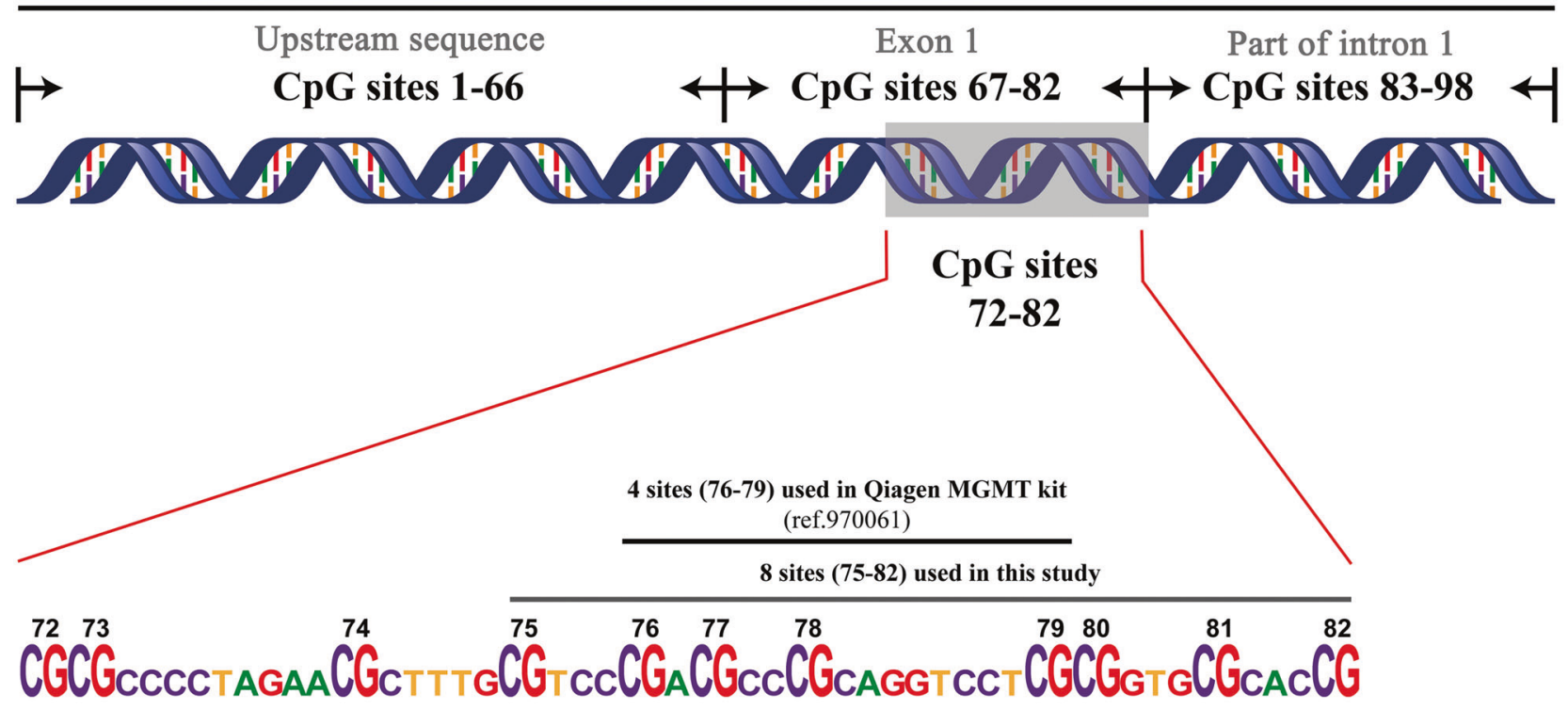

7sites (72-78) used in this study

5 sites (74-78) used in Qiagen MGMT kit
(ref.972032; 970032)

ref.: the item number for Qiagen kits

B

WHO grade

IDH $1 / 2$

Average

CpG site 75

CpG site 76

CpG site 77

CpG site 78

CpG site 79

CpG site 80

$\mathrm{CpG}$ site 81

CpG site 82

impact on $M G M T$ expression and therefore on predicting the response to temozolomide treatment $[11,19,20]$. CpGs 72-82, which are localized in exon 1 , are therefore the most

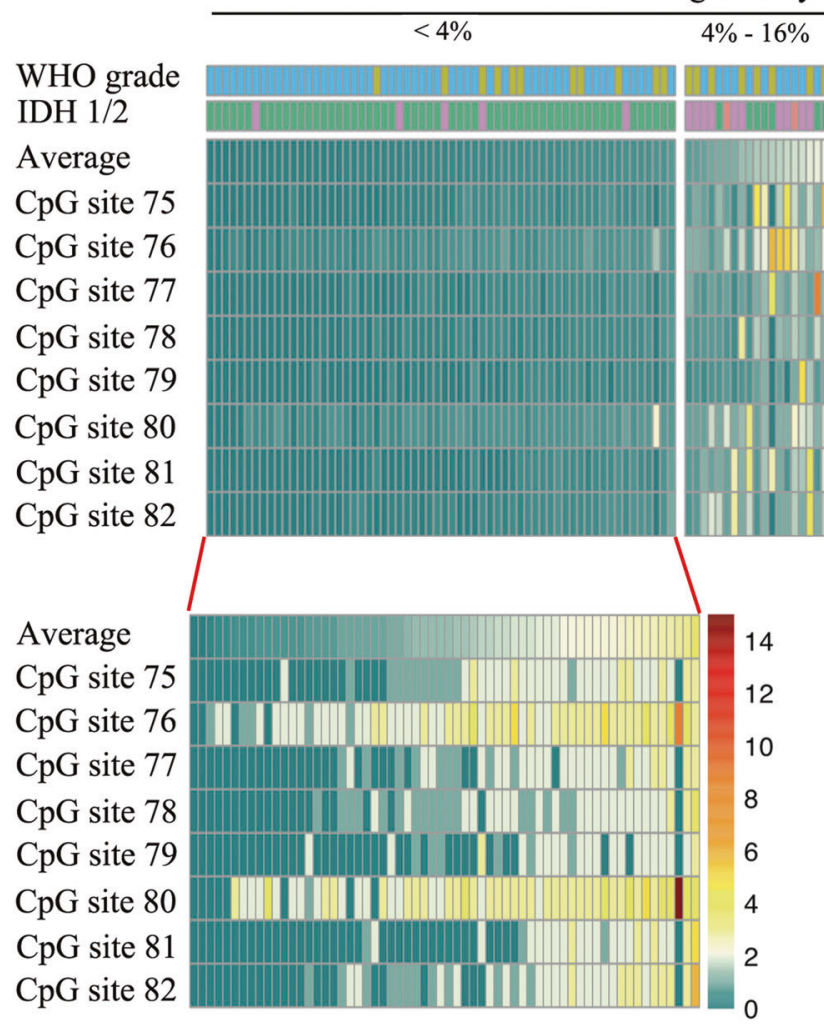

Average methylation level
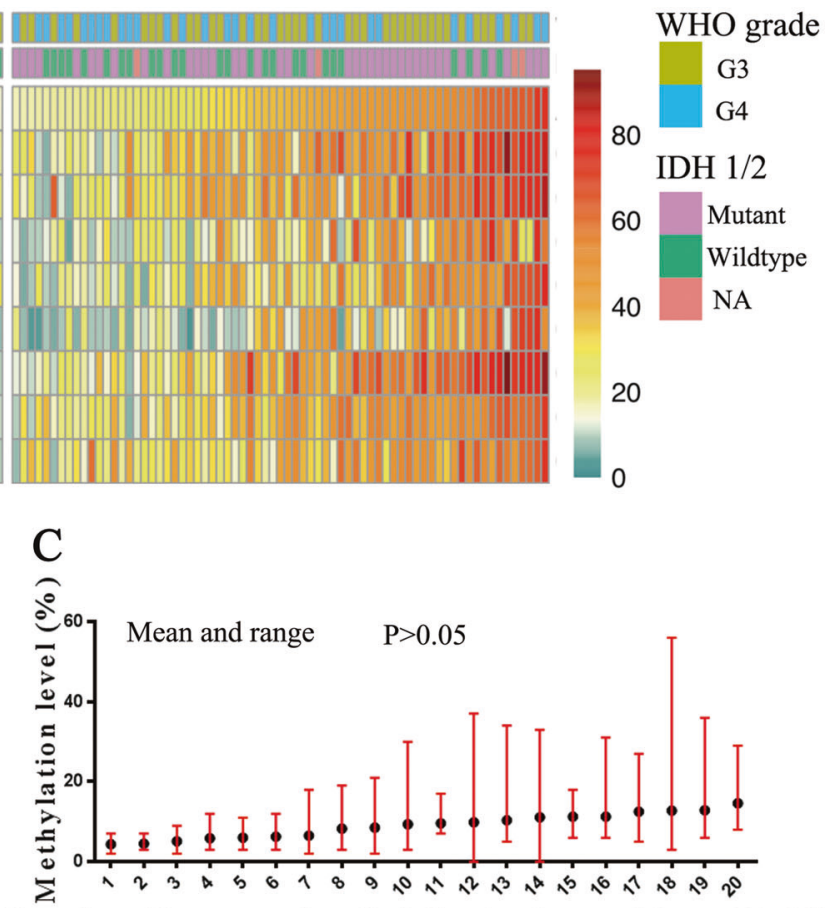

Samples with averaged methylation level ranged from 4\%-16\%

commonly selected $\mathrm{CpGs}$ for pyrosequencing testing [11, 16], while CpGs 74-78 and 76-79 have also been used in commercial kits (Fig. 1). 
Fig. $1 \mathrm{CpG}$ islands and heterogeneity of methylation statuses for each individual $\mathrm{CpG}$ site in the MGMT promoter region. a Map of the distribution of CpGs in the MGMT 5' CpG island. CpGs 72-82, which are most commonly used in pyrosequencing, are shown in the lower panel. The eight and seven CpGs used in this study are indicated by gray and light blue lines, respectively. CpGs 74-78 and 76-79 are indicated by black and dark blue lines, respectively. b Methylation levels of each $\mathrm{CpG}$ were depicted in a heatmap for cohort A. Average refers to the average methylation level of CpGs 75-82. A part of the heatmap (with average methylation level $<4 \%$ ) was displayed again in another heatmap (in the lower left corner) with a more discriminative color bar. c Methylation levels in patients with average methylation levels of $4-16 \%$ were compared. The mean and range were indicated by the black point and red line, respectively

The arithmetic average method, which simply averages the percentage methylation of several consecutive CpGs using a threshold value, has been widely used to interpret pyrosequencing testing results [11, 12, 15-17]. However, this method neglects the potentially large deviations from the average methylation level for individual $\mathrm{CpGs}$, thus reducing the apparent heterogeneity in $\mathrm{CpG}$ methylation levels. In our practice, 10-20\% of patients had methylation levels within $\pm 5 \%$ of the threshold value. Several investigators have noted the existence of a possible "gray zone" resulting from the averaging method, leading to an ambiguous predictive performance [15, 21-23]. The performance of this arithmetic average approach is thus inadequate in clinical practice, especially in the case of methylation levels around the set threshold value. There is therefore a need for a more comprehensive analytical approach to interpreting pyrosequencing results.

In the present study, we determined the heterogeneity of CpG methylation levels in exon 1 of the MGMT gene in two independent cohorts including 213 patients with WHO grade III/IV gliomas. In view of the results, we applied a novel analysis that considered a separate cut-off value for each individual $\mathrm{CpG}$ and confirmed that this approach provided a more useful interpretation of pyrosequencing results for $M G M T$ promoter methylation in gliomas. The superiority of the novel analysis was also validated in an independent cohort of 65 glioma patients.

\section{Materials and methods}

\section{Patients and samples}

A total of 213 patients with WHO grade III/IV gliomas were enrolled from the Chinese Glioma Genome Atlas. $M G M T$ pyrosequencing data for these patients was based on tests of two batches of freshly frozen samples: cohort A (CpGs 75-82; $n=153$ patients) and cohort B (CpGs 72-78; $n=60$ patients). The clinical information for these 213 patients is summarized in Supplementary Table S1. The heterogeneity of different individual MGMT CpG methylation levels was analyzed for all 213 patients.

To assess the predictive value of the MGMT promoter methylation status on the efficacy of the temozolomide chemotherapy, in total 200 patients who underwent temozolomide-based chemotherapy were included into the survival analysis. Among the 200 patients (Table 1), 135 were from the previous 213 patients, and the other 65 were from an additional independent cohort of 65 patients with WHO grade III/IV gliomas of which formalin-fixed paraffin-embedded samples were available.

The chemotherapy protocols for the patients were following the suggestion from the National Comprehensive Cancer Network Clinical Practice Guidelines and the Chinese Glioma Cooperative Group Clinical Practice Guidelines for the management of adult diffuse gliomas [18]. In detail, most of the patients $(77.0 \%)$ received temozolomide treatment following the standard Stupp protocol, during the radiotherapy for 6 weeks, $75 \mathrm{mg}$ temozolomide per square meter of body-surface area per day, 7 days per week; and during the 6 temozolomide treatment cycles, 150-200 mg temozolomide per square meter of body-surface area per day, 5 days during each 28-day cycle. Some of the patients $(18 \%)$ received temozolomide between 3 and 5 cycles, while some of the patients $(4 \%)$ received temozolomide more than 6 cycles. A few of patients $(2 \%)$ received only temozolomide cycles without radiotherapy. The detailed treatment information of patients with gliomas in different subgroups were summarized (Table 2), and the information in each cohort was also listed (Supplementary Table S2).

All specimens were re-evaluated by two independent pathologists and samples with $>80 \%$ tumor cells were used to determined $M G M T$ promotor methylation. The $1 \mathrm{p} / 19 \mathrm{q}$ status of these specimens were determined using dual-color fluorescence in situ hybridization, and the IDHI R132H and IDH2 R172K/M mutations were determined by the DNA pyrosequencing. The characteristics of the test and validated cohorts are summarized in Table 1.

\section{DNA isolation and bisulfite modification}

Genomic DNA was extracted from freshly frozen tumor tissues and formalin-fixed paraffin-embedded samples using a QIAamp DNA Mini Kit (Qiagen, Germany) and QIAamp DNA FFPE Tissue Kit (Qiagen), respectively. The DNA concentration and quantity were tested using a Nano-Drop ND-1000 spectrophotometer (NanoDrop Technologies, Houston, TX, USA), and 100 ng DNA was then used for bisulfite conversion using an Epitect Bisulfite kit (Qiagen) according to the manufacturer's protocol. 
Table 1 Clinicopathological characteristics for patients who received temozolomide

\begin{tabular}{|c|c|c|c|c|c|}
\hline & & \multirow[b]{2}{*}{ Total (200) } & \multicolumn{2}{|c|}{ Frozen tissue } & \multirow{2}{*}{$\begin{array}{l}\text { FFPE samples } \\
\text { The validation } \\
\text { cohort }(65)\end{array}$} \\
\hline & & & $\begin{array}{l}\text { The cohort } \\
\text { A (86) }\end{array}$ & $\begin{array}{l}\text { The cohort } \\
\text { B (49) }\end{array}$ & \\
\hline \multirow[t]{3}{*}{ Age (years) } & Median (range) & $44(17-79)$ & $43(19-72)$ & $46(24-79)$ & $45(17-69)$ \\
\hline & Age $\geq 45$ & $100(50 \%)$ & $41(48 \%)$ & $26(53 \%)$ & $33(51 \%)$ \\
\hline & Age $<45$ & $100(50 \%)$ & $45(52 \%)$ & $23(47 \%)$ & $32(49 \%)$ \\
\hline \multirow[t]{2}{*}{ Gender } & Male & $126(63 \%)$ & $54(63 \%)$ & $33(67 \%)$ & $39(60 \%)$ \\
\hline & Female & $74(37 \%)$ & $32(37 \%)$ & $16(33 \%)$ & $26(40 \%)$ \\
\hline \multirow[t]{3}{*}{ KPS score } & $\mathrm{KPS} \geq 80$ & $103(52 \%)$ & $44(51 \%)$ & $26(53 \%)$ & $33(51 \%)$ \\
\hline & $\mathrm{KPS}<80$ & $87(44 \%)$ & $37(43 \%)$ & $21(43 \%)$ & $29(45 \%)$ \\
\hline & N/A & $10(5 \%)$ & $5(6 \%)$ & $2(4 \%)$ & $3(5 \%)$ \\
\hline \multirow[t]{2}{*}{ WHO grade } & III & $81(41 \%)$ & $35(41 \%)$ & $24(49 \%)$ & $22(34 \%)$ \\
\hline & IV & $119(60 \%)$ & $51(59 \%)$ & $25(51 \%)$ & $43(66 \%)$ \\
\hline \multirow[t]{7}{*}{$\begin{array}{l}\text { WHO } 2016 \\
\text { classification }\end{array}$} & $\begin{array}{l}\text { Anaplastic Oligo, IDH- } \\
\text { mutant, } 1 \mathrm{p} / 19 \mathrm{q} \text { codeletion }\end{array}$ & $8(4 \%)$ & $3(3 \%)$ & $3(6 \%)$ & $2(3 \%)$ \\
\hline & $\begin{array}{l}\text { Anaplastic Astro, IDH- } \\
\text { mutant }\end{array}$ & $34(17 \%)$ & $21(24 \%)$ & $8(16 \%)$ & $5(8 \%)$ \\
\hline & $\begin{array}{l}\text { Anaplastic Astro, IDH- } \\
\text { wildtype }\end{array}$ & $30(15 \%)$ & $11(13 \%)$ & $13(27 \%)$ & $6(9 \%)$ \\
\hline & $\begin{array}{l}\text { Glioblastoma, IDH- } \\
\text { mutant }\end{array}$ & $28(14 \%)$ & $11(13 \%)$ & $6(12 \%)$ & $11(17 \%)$ \\
\hline & $\begin{array}{l}\text { Glioblastoma, IDH- } \\
\text { wildtype }\end{array}$ & $84(42 \%)$ & $36(42 \%)$ & $18(37 \%)$ & $30(46 \%)$ \\
\hline & Anaplastic Astro, NOS a & $9(5 \%)$ & $2(2 \%)$ & $0(0 \%)$ & $7(0 \%)$ \\
\hline & Glioblastoma, NOS ${ }^{\mathrm{a}}$ & $7(4 \%)$ & $2(2 \%)$ & $1(2 \%)$ & $4(17 \%)$ \\
\hline \multirow[t]{2}{*}{ 1p19q codeletion } & Codel & $8(4 \%)$ & $3(3 \%)$ & $3(6 \%)$ & $2(3 \%)$ \\
\hline & Non-codel & $192(96 \%)$ & $83(97 \%)$ & $46(94 \%)$ & $63(97 \%)$ \\
\hline \multirow[t]{3}{*}{ IDH1/2 mutation } & Mutation & $70(35 \%)$ & $35(41 \%)$ & $17(35 \%)$ & $18(28 \%)$ \\
\hline & Wildtype & $114(57 \%)$ & $47(55 \%)$ & $31(63 \%)$ & $36(55 \%)$ \\
\hline & N/A & $16(8 \%)$ & $4(5 \%)$ & $1(2 \%)$ & $11(17 \%)$ \\
\hline
\end{tabular}

Oligo oligodendroglioma, Astro astrocytoma

${ }^{a}$ NOS refers to the $I D H$ status is lacking. And the $1 \mathrm{p} / 19 \mathrm{q}$ of these gliomas are non-codeletion in this study

\section{Pyrosequencing of MGMT promoter}

The MGMT pyrosequencing template was prepared as described previously [12, 24]. Briefly, bisulfite-treated DNA was amplified using the forward primer 5'-GTT TYG GAT ATG TTG GGA TAG TT-3' and biotinylated reverse primer 5'-biotin-ACR ACC CAA ACA CTC ACC AA-3'. Two independent assays were performed in different samples with two pyrosequencing sequencing primers: $5^{\prime}-$ GAT ATG TTG GGA TAG T-3' (for CpGs 72-78) and 5'GTT TTT AGA AYG TTT TG-3' (for CpGs 75-82). The analyzed sequences for CpGs 72-78 and 75-82 were TYG YGT TTT TAG AAY GTT TTG YGT TTY GAY GTT YGT AGG T and YGT TTT GYG TTT YGA YGT TYG TAG GTT TTY GYG GTG YGT A, respectively. Pyrosequencing was performed using a PyroMarker Q96 instrument and the results were analyzed with PyroMarker Q96 software (Qiagen). The formalin-fixed paraffinembedded samples from the 65 patients in the validation cohort were tested using a PyroMarker Q24 instrument with pyrosequencing primers: 5'-GAT ATG TTG GGA TAG TT-3' (for CpGs 72-78), and the analyzed sequence was YGY GTT TTT AGA AYG TTT TGY GTT TYG AYG TTY GT.

\section{Methylation-specific PCR}

Bisulfite-treated DNA was amplified with SYBR $^{\circ}$ Select Master Mix (Thermo Fisher, Waltham, MA, USA). The sequences of the methylated primers were 5'-TTT CGACGTTCGTAGGTTTTCGC-3' (forward primer) and 5' '-GCACTCTTCCGAAAACGAAACG-3' (reverse primer), and the sequences of the unmethylated primers were 5'-TTTGTGTTTTGATGTTTGTAGGTTTTTGT-3' (forward primer) and 5'-AАCTCCACACTCTTCCAAAAACA AAACA-3' (reverse primer). The annealing temperature was $59^{\circ} \mathrm{C}$. The results were analyzed with ABI 7500 software (Thermo Fisher). Unmethylated DNA and commercial 


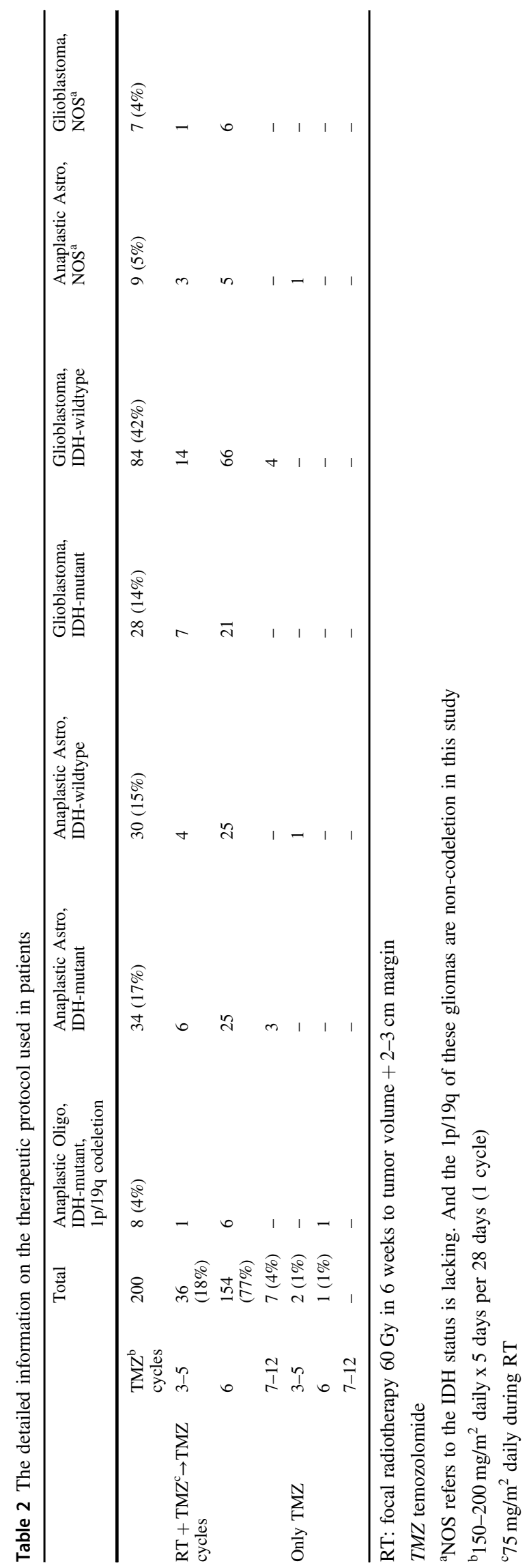

methylated DNA (Qiagen) were used as controls in each set of reactions. In addition to analyzing the methylation status by comparing the melting curves and $\mathrm{Ct}$ values with the controls, the reaction products were also checked by $2.5 \%$ agarose gel electrophoresis to verify the products with lengths $81 \mathrm{bp}$ (methylated) and $93 \mathrm{bp}$ (unmethylated), respectively.

\section{Statistical analysis}

NThe analytical strategy of the present study is shown in Supplementary Figure S1. The patients were stratified based on the median overall survival (17 months) according to receiver operating characteristic curve analysis. The calculated area under the curve, $P$-value, sensitivity, specificity, positive likelihood ratio (sensitivity / 1-specificity), and Youden's factor (sensitivity + specificity -1 ) were used to evaluate the predictive value of $M G M T$ methylation according to the different analytical models based on the methylation levels of individual and consecutive tested CpGs.

Consecutive $\mathrm{CpG}$ weighted average methylation levels were calculated using the following formula [25]:

$$
\text { Weighted average }=\frac{\sum_{i}^{\beta} * s i t_{i}}{\sum_{i}^{\beta}} \text {, where the coefficient } \beta \text { for }
$$

each individual $\mathrm{CpG}$ methylation level was calculated using univariate Cox analysis [26].

Univariate and multivariate survival analyses were performed using the Cox regression model, and survival curves were estimated by Kaplan-Meier curves and compared using the log-rank test. Methylation levels were compared among different individual CpGs or different patients by one-way analysis of variance with the Kruskal-Wallis test. Multiple comparisons were performed using Dunn's multiple comparison tests. All statistical analyses were performed using SPSS software (IBM, NY, USA) and GraphPad Prism 7 (GraphPad Software, CA, USA).

\section{Results}

\section{Heterogeneity among CpG methylation levels in MGMT promoter}

Some of the 98 individual CpGs in the MGMT gene promoter are shown in Fig. 1a. CpGs 72-82, localized in exon 1 , are the most commonly used sites for pyrosequencing testing, while CpGs 74-78 and 76-79 have also been used in commercial kits. We tested CpGs $72-78$ and $75-82$ in cohorts A and B, respectively (Fig. 1a), and CpGs 72-78 in the independent validation set. 
Table 3 Optimal cut-off value for each individual $\mathrm{CpG}$

\begin{tabular}{|c|c|c|c|c|c|c|c|c|}
\hline \multirow[t]{2}{*}{ CpG sites } & \multirow[t]{2}{*}{ Cut-off value } & \multirow[t]{2}{*}{ AUC } & \multirow[t]{2}{*}{ Standard error } & \multirow[t]{2}{*}{$\mathrm{P}$} & \multicolumn{2}{|c|}{$\begin{array}{l}95 \% \\
\text { confidence } \\
\text { interval }\end{array}$} & \multirow[t]{2}{*}{ Sensitivity } & \multirow[t]{2}{*}{ Specificity } \\
\hline & & & & & Lower & Upper & & \\
\hline 75 & 16 & 0.693 & 0.060 & 0.002 & 0.576 & 0.809 & $71 \%$ & $68 \%$ \\
\hline 78 & 4 & 0.682 & 0.060 & 0.004 & 0.566 & 0.799 & $76 \%$ & $60 \%$ \\
\hline 81 & 11 & 0.678 & 0.060 & 0.006 & 0.560 & 0.797 & $68 \%$ & $68 \%$ \\
\hline 82 & 15 & 0.674 & 0.061 & 0.007 & 0.554 & 0.793 & $65 \%$ & $70 \%$ \\
\hline 76 & 12 & 0.673 & 0.060 & 0.007 & 0.555 & 0.791 & $71 \%$ & $64 \%$ \\
\hline 77 & 10 & 0.659 & 0.062 & 0.013 & 0.538 & 0.781 & $56 \%$ & $76 \%$ \\
\hline 80 & 15 & 0.659 & 0.062 & 0.014 & 0.538 & 0.780 & $62 \%$ & $70 \%$ \\
\hline 79 & 5 & 0.644 & 0.062 & 0.026 & 0.522 & 0.765 & $65 \%$ & $64 \%$ \\
\hline
\end{tabular}

$A U C$ area under the curve of the receiver operating characteristic curve analysis
$\mathbf{A}$

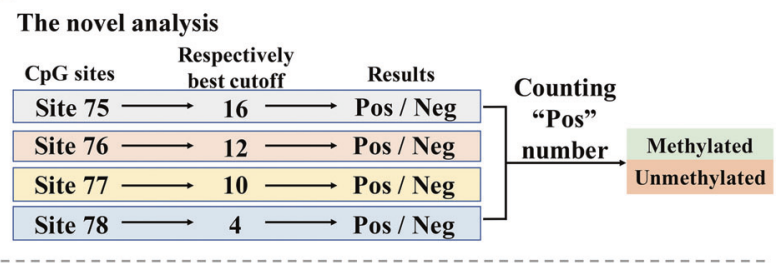

Traditional analysis according to average methylation level

CpG sites
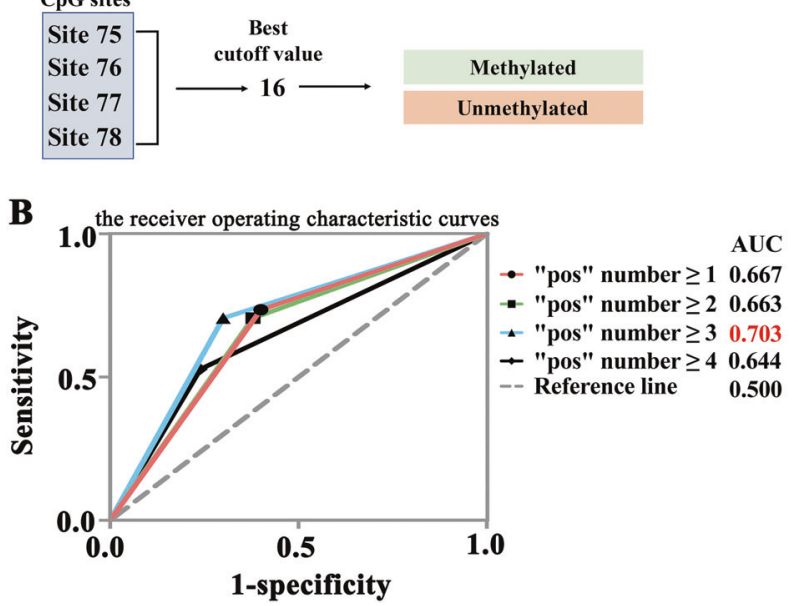

Fig. 2 Comparison of the novel analytical and traditional arithmetic average methods. a Novel analytical and traditional average methods for CpGs 75-78. The respective optimal cut-off values are calculated in Table 2. The cut-off for the novel model was determined by the positive (Pos) and negative (Neg) numbers of selected CpGs. b The best cut-off for the novel analytical model was calculated by the receiver operating characteristic curve analysis. AUC, area under the curve

To gather a general idea of the methylation levels of each individual $\mathrm{CpG}$ among the gliomas, we presented the methylation levels for each individual $\mathrm{CpG}$ in cohort $\mathrm{A}$ (Fig. 1b) and cohort B (Supplementary Figure S2A) as heatmaps. Most previous studies used cut-off values of $4-16 \%$ to determine the final methylation status [11-17], and we therefore separated the heatmaps into three parts according to average methylation levels of $4 \%$ and $16 \%$. High degrees of heterogeneity were observed among MGMT promoter CpGs in all three parts, and individual CpG methylation levels showed high standard deviations in most samples. There was consequently no significant difference in $\mathrm{CpG}$ methylation levels between any two samples with average methylation levels of $4-16 \%$ in either tested cohort (Fig. 1c, Supplementary Figure S2B).

\section{Distinct predictive effect of each individual CpG methylation level}

The heterogeneity among different individual CpGs indicated their potentially different predictive effects. This hypothesis was confirmed by the receiver operating characteristic curve analysis for each individual $\mathrm{CpG}$ of $\mathrm{CpGs}$ 75-82 in cohort A. All the individual CpGs could predict survival, but the optimal cut-off values for each individual CpG varied from 4\%-16\% (Table 3). Similar results were observed for CpGs 72-78 in cohort B (data not shown).

\section{Advantages of the novel analytical model for MGMT pyrosequencing}

The high methylation heterogeneity of MGMT promoter CpGs suggested that the arithmetic average method maybe not be ideal for determining the final MGMT methylation status. We therefore developed a novel analytical model that defined the final MGMT methylation status with reference to the methylation statuses of each individual $\mathrm{CpG}$, with their own cut-off values. For convenience and to improve on the traditional average method with minimal change, our novel analysis also utilized groups of several consecutive CpGs. The analytical processes for the novel and traditional average methods were demonstrated using CpGs 75-78 in cohort A (Fig. 2a). CpGs 75-78 showed the best predictive accuracy among all four-consecutive CpGs in cohort A 


\section{The cohort A}
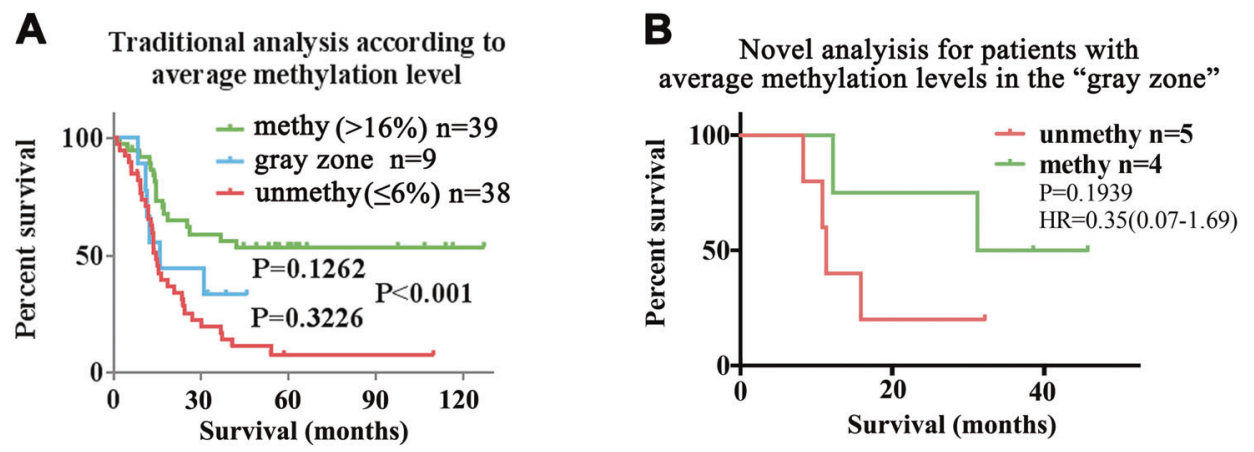

The cohort B
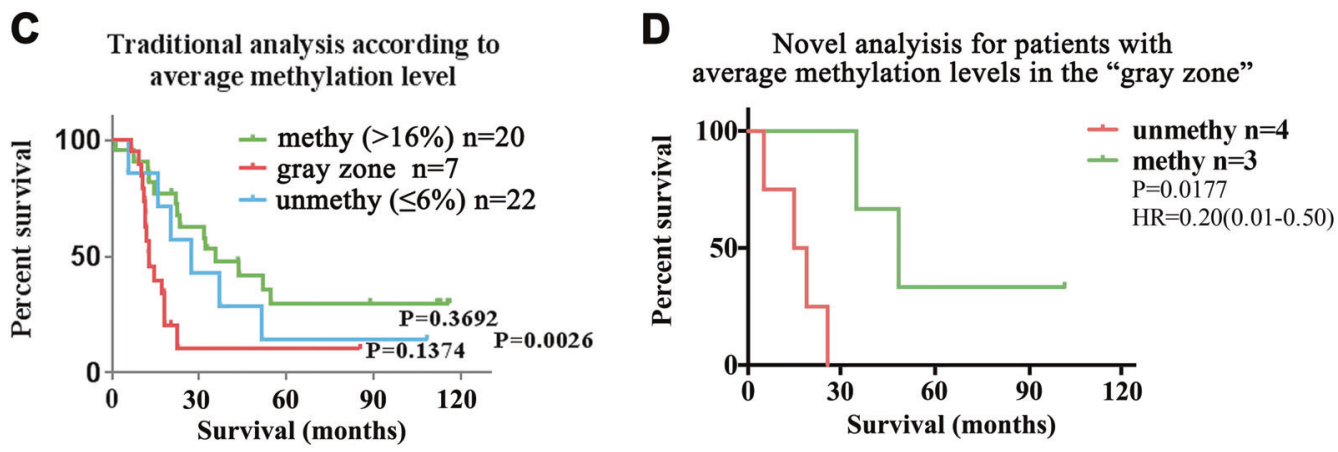

The validation cohort

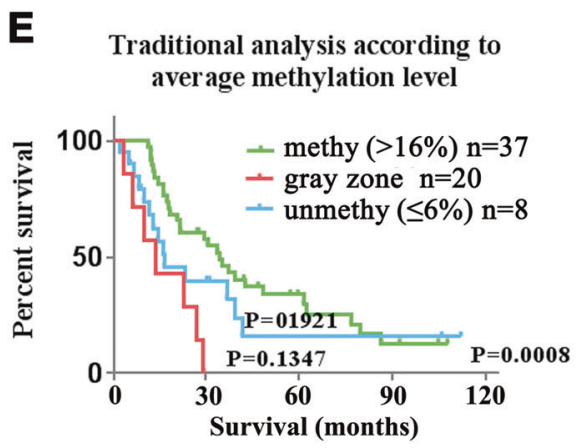

Fig. 3 Practicability of the novel analytical model in patients with methylation levels in the "gray zone" according to the traditional average approach. a-f Kaplan-Meier survival curves for patients

according to the traditional average method (Supplementary Table S3). The optimal number of "positive methylation counts" for determining the methylation status in the novel analytical model was determined by receiver operating characteristic curve analysis, and the best predictive effect occurred using a positive methylation count $\geq 3$ as the judgment criterion (Fig. 2b). According to this criterion, the novel model could distinguish the prognosis of patients with "gray zone" methylation status (average methylation levels of 6-16\%) according to the traditional analysis in all three cohorts (Figs. 3a-f).

In addition to comparing the novel analysis with the traditional average method, we also compared its predictive
F Novel analyisis for patients with average methylation levels in the "gray zone"

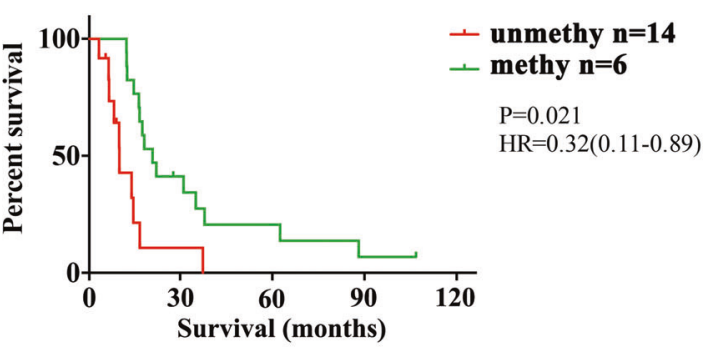

stratified by methylation status of CpGs $75-78$ based on the average and novel analytical approaches. $P$ values were calculated by log-rank tests. methy, methylated; unmethy, unmethylated. $H R$ hazard ratio

accuracy with the weighted average analytical approach, which weighted each $\mathrm{CpG}$ with a factor calculated by univariate Cox regression analysis (Supplementary Table S4). The predictive accuracy of the novel analytical model was superior according to the area under the curves of the receiver operating characteristic curve analysis. We therefore focused on comparing the novel and traditional average methods in the following investigations.

We compared the predictive values of the novel and traditional average analytical methods in cohort A (Fig. 4). Although the difference was marginal, the novel approach showed a better predictive performance in terms of overall survival in Kaplan-Meier curves (Fig. 4a). Of note, four 
The cohort A
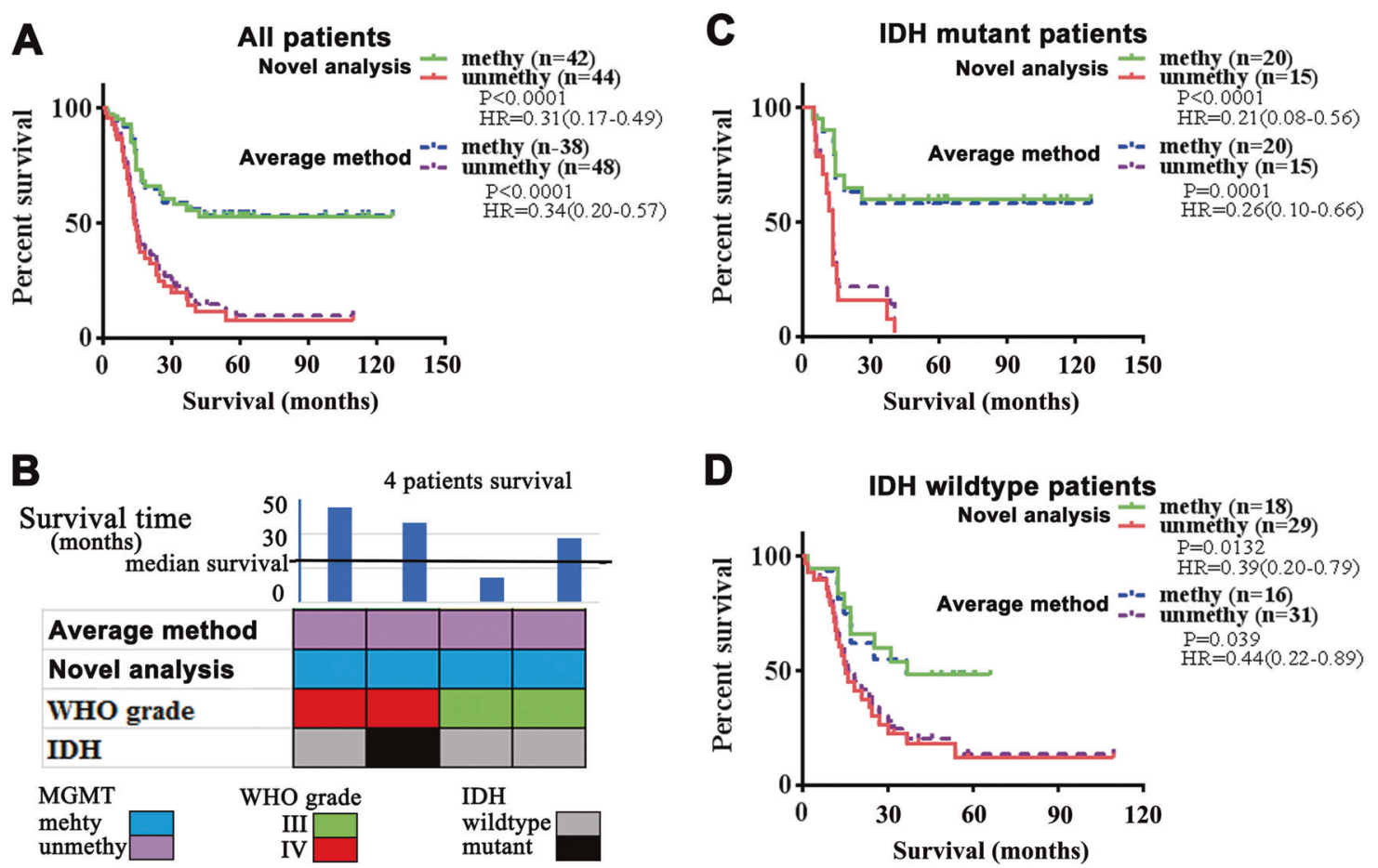

Fig. 4 Superiority of the novel analytical model in cohort A. a Kaplan-Meier curves for patients stratified according to the novel and average methods for CpGs 75-78. b Clinicopathological features of patients with discrepant results between the novel and average

patients, three of who survived longer than the median overall survival (17 months), were regarded as unmethylated according to traditional analysis but as methylated according to the novel analysis (Fig. 4b). Given the potential influences of $I D H$ status on evaluating the therapeutic prognostic value of $M G M T$ methylation [27, 28], we also evaluated the novel analytical model in patients stratified by $I D H$ status. The predictive performance of the novel approach was better than that of the traditional analysis in patients with either mutant (Fig. 4c) or wild-type IDH (Fig. 4d). Considering the differences among gliomas in different subgroups according to the WHO 2016 Classification [29], we evaluated the novel analytical model in patients with glioblastoma, IDH-wildtype subgroup, and the predictive performance of the novel approach was also better than that of the traditional analysis (Supplementary Figure S3A). But we were unable to compare the methods in other subgroups because of the limited patient numbers. Meanwhile, we also observed that the methylation status of $M G M T$ promoter by this novel analysis could not predict the prognosis in patients not receiving alkylating chemotherapy in the glioblastomas or all high-grade gliomas (Supplementary Figure S4).

We also evaluated the usability of the novel analytical model for CpGs 75-78 in cohort B (Supplementary

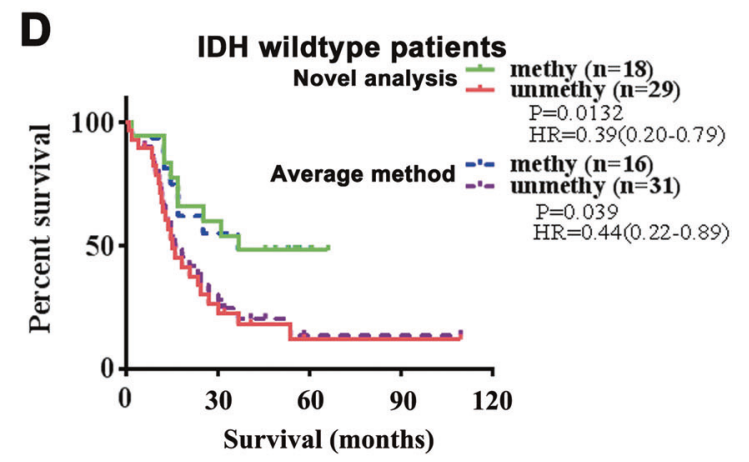

approaches. c, d Kaplan-Meier curves for patients with $I D H$ mutant (c) and $I D H$ wild-type statuses (d) stratified according to the novel and average methods for CpGs 75-78. HR hazard ratio

Figure S5), using the same criteria as in cohort A. We calculated the area under the curves of the receiver operating characteristic curve analysis for both methods. The area under the curve for the novel analysis (hazard ratio, 0.836; 95\% confidence interval $0.715-0.958$ ) was greater than that for the average method (hazard ratio, $0.761 ; 95 \%$ confidence interval 0.617-0.905) (Supplementary Figure S5A), as confirmed by Kaplan-Meier analysis (Supplementary Figure S5B). In the four patients with discrepant methylation statuses noted above, the methylation status determined by the novel approach was better correlated to the patient's prognosis (Supplementary Figure S5C). The novel analytical model was also superior to the average method in $I D H$ wildtype patients (Supplementary Figure S5D).

Finally, we performed univariate and multivariate Cox regression analyses. In univariate analysis, WHO grade $(P$ $<0.001), M G M T$ status determined by the novel analytical model $(P=0.002)$, and $I D H$ status $(P=0.003)$ were significantly correlated with prognosis, while $M G M T$ status determined by the novel approach remained an independent prognostic factor in multivariate analysis $(P=0.009)$ (Supplementary Table S5).

Overall, MGMT status defined using the novel analytical approach provided a more accurate predictive value compared with the traditional arithmetic average approach. 
The validation cohort

A

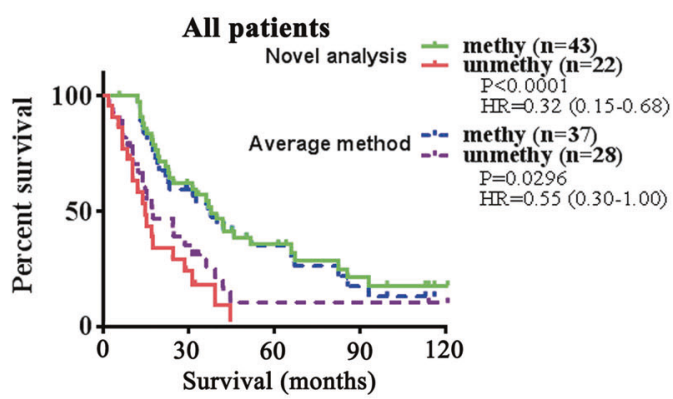

B

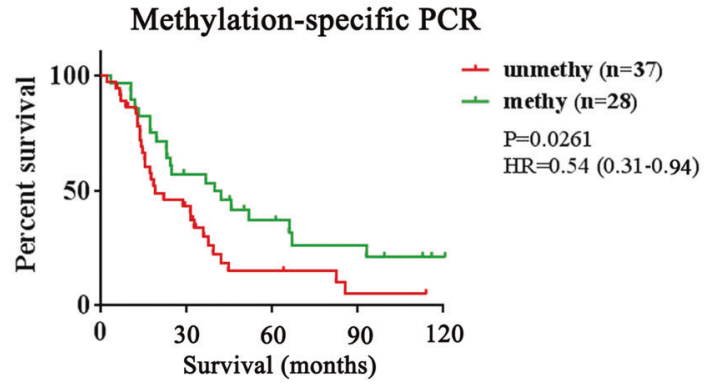

C The receiver operating

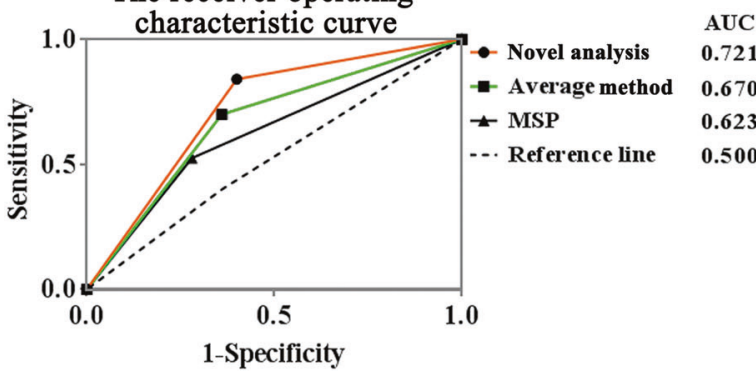

Fig. 5 Validation of the advantages of the novel analytical model in the validation cohort. a, b Survival analysis of 65 patients stratified according to the novel analytical model for CpGs 75-78 (a), average method for CpGs 75-78 (a), and Methylation-specific PCR detection results (b). c Predictive accuracies of the novel and average methods,

\section{Validation of the novel analytical model in an independent cohort}

We further confirmed the advantages of the novel analytical model for MGMT pyrosequencing by comparing the predictive accuracies of the different methods in formalin-fixed paraffin-embedded samples from 65 patients with WHO III/ IV gliomas, and also with methylation-specific PCR testing. Kaplan-Meier curves showed that the predictive performance of the novel method (hazard ratio, 0.32; 95\% confidence interval, $0.150-0.680$ ) was better than that of the average method (hazard ratio, $0.55 ; 95 \%$ confidence interval, 0.300-1.000) and of methylation-specific PCR testing (hazard ratio, $0.54 ; 95 \%$ confidence interval $0.310-0.940$ ) (Figs. 5a, b). The area under the curve of the receiver operating characteristic curve analysis for the novel analysis $(0.721,95 \%$ confidence interval $0.588-0.853)$ was greater
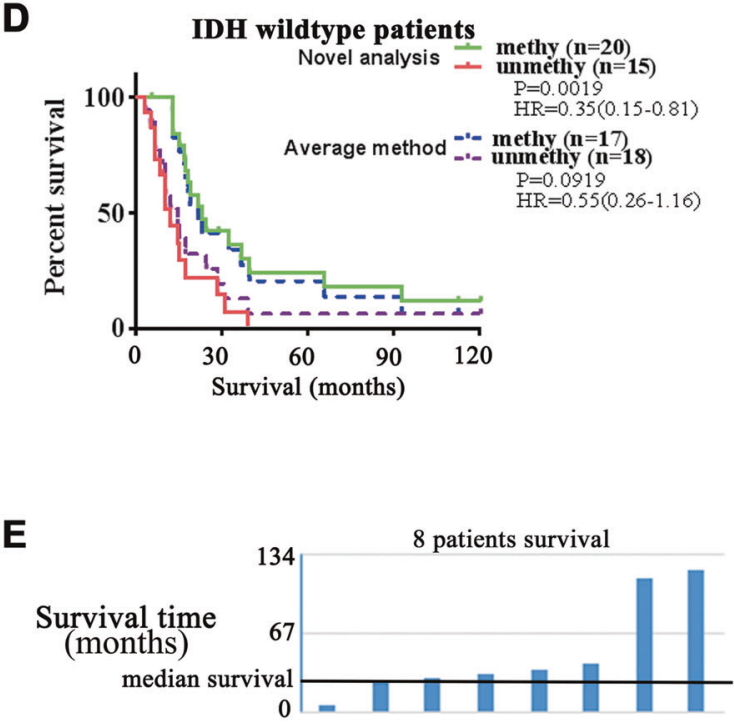

\begin{tabular}{l|l|l|l|l|l|l|l|l|}
\hline $\begin{array}{l}\text { Average method } \\
\text { Novel analysis }\end{array}$ & & & & & & & \\
\hline WHO grade & & & & & & & \\
\hline IDH & & & & & \\
$\begin{array}{l}\text { MGMT } \\
\text { mehty } \\
\text { unmethy }\end{array}$ \\
\hline
\end{tabular}

and Methylation-specific PCR testing (d) were evaluated by the receiver operating characteristic curve analysis. $A U C$ area under the curve. e Survival and pathological characteristics of the patients with discrepant results between the novel and average analytical methods. $\mathrm{HR}$, hazard ratio

than that for the average method $(0.670,95 \%$ confidence interval 0.533-0.807) and for methylation-specific PCR testing $(0.623$, 95\% confidence interval 0.483-0.762) (Fig. 5c). Moreover, we also validated the superiority of the novel analysis in $I D H$ wild-type patients (Fig. 5d) and patients with glioblastoma, IDH-wildtype subgroup (Supplementary Figure S3B). Methylation status determined by the novel approach was also better correlated with prognosis in eight patients with discrepant results between the two methods (Fig. 5e)

\section{Performance of the novel analytical model in commercial kits}

CpGs 76-79 (named "Kit 1") and CpGs 74-78 (“Kit 2") have been used in Qiagen commercial kits (Fig. 1). Kit 1 was included in the cohort A CpGs (75-82) and Kit 2 was 
included in the cohort B CpGs (72-78). In Kit 1, the area under the curve for the novel model $(0.685,95 \%$ confidence interval $0.570-0.780$ ) was greater than that for the arithmetic average approach $(0.668,95 \%$ confidence interval $0.550-0.786$ ). The hazard ratio according to Kaplan-Meier for the novel analysis $(0.330,95 \%$ confidence interval 0.193-0.563) was also better than that for the average approach $(0.361,95 \%$ confidence interval 0.184-0.555). Similar results were observed for Kit 2 (Supplementary Figure S6).

\section{Discussion}

Quantitative MGMT pyrosequencing results are often dichotomized based on the arithmetic average of several tested CpGs and a threshold value [12, 16, 30, 31]. However, this traditional averaging method involves some uncertainty when the average methylation value is close to the threshold value (gray zone), which results from neglecting the high heterogeneity among individual CpGs $[3,22]$. In the present study, we applied a novel analytical model to comprehensively evaluate the methylation status of each selected $\mathrm{CpG}$ according to its own cut-off value. This novel approach defined MGMT methylation as occurring when at least three $\mathrm{CpGs}$ exceeded the respective threshold value determined from the receiver operating characteristic curve analysis. Our results demonstrated that this novel analytical model of $M G M T$ pyrosequencing could predict the therapeutic efficacy of patients with glioma more accurately than the traditional average method, and was also superior to methylation-specific PCR testing. The advantage of the novel model was particularly evident in patients with 'gray zone' results according to the traditional average approach.

Currently, several methods for MGMT promoter methylation testing have been reported, including gel-based methylation-specific PCR [32], methylation-specific quantitative PCR [33], methylation-specific quantitative PCR plus specific probe, MethyLight quantitative PCR [7], Methylation-sensitive high resolution melting [34], pyrosequencing [9], Methylation-specific, multiplex ligationdependent probe amplification [35] and microarray chips, e.g. Illumina methylation array with HM- $450 \mathrm{~K}$ or HM-27K chips [36-38]. Among these methods, Methylation-specific, multiplex ligation-dependent probe amplification is the only method not requiring bisulfite treatment, but usually it can only detect the methylation level of one $\mathrm{CpG}$ site, which is also limited to the restriction sites [23]. Methylation-specific PCR is the first method that has been repeatedly shown to be of predictive or prognostic value in clinical trials $[1,39]$. Methylation-specific PCR is also the basis of other PCRbased methods, including methylation-specific quantitative
PCR, MethyLight quantitative PCR and Methylationsensitive high-resolution melting. Though Methylationspecific PCR and Methylation-specific PCR-derived methods are easy to perform, they can only recognize fully methylated or unmethylated sequences with specially designed methylation - specific primers or probes, and each primer or probe typically interrogates a series of 3 to $5 \mathrm{CpG}$ sites $[9,32,33]$. Given the heterogeneity of the methylation levels of $\mathrm{CpG}$ sites in the MGMT promoter [11], Methylation-specific PCR based methods have the drawbacks to reflect the heterogeneity of $\mathrm{CpG}$ sites [23]. Moreover, the results of these methods critically depend on the employed PCR protocols [15], and thus lacking stability [40]. The method with microarray chips is usually utilized in large-scale analyses of the methylome of gliomas, it is inconvenient to be used in clinical testing, due to the high cost and complicated data analysis process [11,38].

Pyrosequencing is a robust technique, which could assess the methylation percentage of all individual CpGs measured by quantification of each added nucleotide during sequencing, and has the ability to evaluate the bisulfite conversion efficiency through internal control $[11,23]$. In this study, we confirmed the predictive performance of pyrosequencing is better than Methylation-specific PCR. The superiority of pyrosequencing had also been demonstrated by two studies comparing others methods in glioblastoma patients [9, 41]. Thus, pyrosequencing, which could offer valid, reliable and quick evaluation of the $M G M T$ promoter methylation status from both formalin fixed and paraffin-embedded specimens, was thought as the "gold standard" for MGMT promoter methylation testing [15, 17, 42].

The CpGs selection and cut-off value determination are the two critical factors that determine the final methylation result of pyrosequencing testing [11, 23]. In a recent study, we confirmed that combinations of four or more CpGs methylation within CpGs 72-82 in MGMT promoter present equivalent predictive value for $M G M T$ expression and temozolomide therapeutic outcomes in gliomas [42]. However, the cut-off value determination is still a pending critical issue for pyrosequencing testing with traditional average analysis method $[21,22]$. Here, the novel analytical model could provide improved solution for the cut-off value determination in pyrosequencing testing. Importantly, there was a clear tendency for the novel analysis to show greater predictive accuracy in both the tested cohorts with fresh frozen tissues and the independent validation cohort with formalin-fixed paraffin-embedded samples. Overall, the proposed novel analytical model of $M G M T$ pyrosequencing offered an optimal predictive performance in patients with WHO grade III/IV gliomas.

A retrospective analysis of the NOA-04 trial revealed that the prognostic and predictive values of $M G M T$ methylation depended on $I D H$ status. It also indicated that 
MGMT was a prognostic factor in patients with $I D H 1 / 2$ mutant gliomas, and predicted the benefit of alkylating chemotherapy in patients with $I D H 1 / 2$ wild-type tumors [27]. Given that the clinical course, prognosis, and mechanism of MGMT methylation may differ between $I D H$-mutated and wild-type gliomas [28], we evaluated the performances of the novel and traditional average methods in patients according to specific $I D H$ status, and demonstrated superiority of the novel approach irrespective of $I D H$ mutation status.

Loss of one copy of the chromosomal region containing the MGMT gene (10q26) is quite common in glioblastoma $(60-90 \%)[37,43]$. It has been reported that there is a negative correlation between MGMT promoter methylation and MGMT expression in glioblastoma with chromosome $10 / 10 \mathrm{q}$ deletion, and there is no significant interaction between MGMT promoter methylation and chromosome $10 / 10 q$ deletion $[37,41]$. Therefore, we did not involve copy number alteration for the chromosome $10 / 10 \mathrm{q}$ in this study. However, it is still an important issue in future study that whether chromosome 10 / 10q copy number alterations might be with prognostic or predictive values concerning response to TMZ treatment through affecting MGMT expression in GBM. Because it has been reported that patients with glioblastoma harboring chromosome $10 / 10 \mathrm{q}$ deletion could benefit more from temozolomide treatment than other glioblastoma patients [38, 43, 44]. Although chromosome $10 / 10 \mathrm{q}$ deletion does not significantly affect MGMT expression of glioblastoma in the TCGA dataset [37].

This study was limited by its retrospective nature and the relatively small population size, and the optimal cut-off value for each $\mathrm{CpG}$ may thus need to be adjusted when applying this novel analytical model. Nevertheless, this study confirmed the superiority of the novel approach to analyzing $M G M T$ pyrosequencing results for predicting the prognosis of glioma patients using either fresh frozen or formalin-fixed paraffin-embedded tissue samples.

In conclusion, the novel analytical approach for pyrosequencing testing has three advantages over the traditional arithmetic average method: it accounts for heterogeneity among individual CpGs in the MGMT promoter region, it provides more accurate results in cases where the methylation level according to the average method is in the 'gray zone', and it shows a better clinical predictive performance for alkylating agent chemotherapy.

Acknowledgements We thank Susan Furness, PhD, from Liwen Bianji, Edanz Group China (www.liwenbianji.cn/ac), for editing the English text of a draft of this manuscript.

Funding This work was supported by the National Natural Science Foundation of China (81773208, 81402052); the Beijing Nova Program (Z16110004916082); the National Key Research and
Development Plan (2016YFC0902500); the Beijing Scienceand Technology Plan (Z131100006113018, Z141100000214009); and the Capital Medical Development Research Fund (2016-1-1072).

\section{Compliance with ethical standards}

Ethics approval and consent to participate This study was approved by the Beijing Tiantan Hospital institutional review board, and informed consent was obtained from each patient involved in our research.

Conflict of interest The authors declare that they have no conflict of interest.

\section{References}

1. Hegi ME, Diserens AC, Godard S, et al. Clinical trial substantiates the predictive value of O-6-methylguanine-DNA methyltransferase promoter methylation in glioblastoma patients treated with temozolomide. Clin Cancer Res. 2004;10:1871-4.

2. Hegi ME, Diserens AC, Gorlia T, et al. MGMT gene silencing and benefit from temozolomide in glioblastoma. $\mathrm{N}$ Engl $\mathrm{J}$ Med. 2005;352:997-1003.

3. Barault L, Amatu A, Bleeker FE, et al. Digital PCR quantification of MGMT methylation refines prediction of clinical benefit from alkylating agents in glioblastoma and metastatic colorectal cancer. Ann Oncol. 2015;26:1994-9.

4. Lechapt-Zalcman E, Levallet G, Dugue AE, et al. O(6) -methylguanine-DNA methyltransferase (MGMT) promoter methylation and low MGMT-encoded protein expression as prognostic markers in glioblastoma patients treated with biodegradable carmustine wafer implants after initial surgery followed by radiotherapy with concomitant and adjuvant temozolomide. Cancer. 2012;118:4545-54.

5. Boots-Sprenger SH, Sijben A, Rijntjes J, et al. Significance of complete $1 \mathrm{p} / 19 \mathrm{q}$ co-deletion, IDH1 mutation and MGMT promoter methylation in gliomas: use with caution. Mod Pathol. 2013;26:922-9.

6. Esteller M, Garcia-Foncillas J, Andion E, et al. Inactivation of the DNA-repair gene MGMT and the clinical response of gliomas to alkylating agents. N Engl J Med. 2000;343:1350-4.

7. Eads CA, Danenberg KD, Kawakami K, et al. MethyLight: a high-throughput assay to measure DNA methylation. Nucleic Acids Res. 2000;28:E32.

8. Gerson SL. MGMT: its role in cancer aetiology and cancer therapeutics. Nat Rev Cancer. 2004;4:296-307.

9. Quillien V, Lavenu A, Karayan-Tapon L, et al. Comparative assessment of 5 methods (methylation-specific polymerase chain reaction, MethyLight, pyrosequencing, methylation-sensitive high-resolution melting, and immunohistochemistry) to analyze O6-methylguanine-DNA-methyltranferase in a series of 100 glioblastoma patients. Cancer. 2012;118:4201-11.

10. Komine C, Watanabe T, Katayama Y, et al. Promoter hypermethylation of the DNA repair gene O6-methylguanine-DNA methyltransferase is an independent predictor of shortened progression free survival in patients with low-grade diffuse astrocytomas. Brain Pathol. 2003;13:176-84.

11. Bienkowski M, Berghoff AS, Marosi C, et al. Clinical Neuropathology practice guide 5-2015: MGMT methylation pyrosequencing in glioblastoma: unresolved issues and open questions. Clin Neuropathol. 2015;34:250-7.

12. Yuan G, Niu L, Zhang Y, et al. Defining optimal cutoff value of MGMT promoter methylation by ROC analysis for clinical setting in glioblastoma patients. J Neurooncol. 2017;133:193-201. 
13. Cheng W, Ren X, Cai J, et al. A five-miRNA signature with prognostic and predictive value for MGMT promoter-methylated glioblastoma patients. Oncotarget. 2015;6:29285-95.

14. Rapkins RW, Wang F, Nguyen HN, et al. The MGMT promoter SNP rs16906252 is a risk factor for MGMT methylation in glioblastoma and is predictive of response to temozolomide. Neuro Oncol. 2015;17:1589-98.

15. Wick W, Weller M, van den Bent M, et al. MGMT testing--the challenges for biomarker-based glioma treatment. Nat Rev Neurol. 2014;10:372-85.

16. Quillien V, Lavenu A, Sanson M, et al. Outcome-based determination of optimal pyrosequencing assay for MGMT methylation detection in glioblastoma patients. $\mathrm{J}$ Neurooncol. 2014;116:487-96.

17. Zhao H, Wang S, Song C, Zha Y, Li L. The prognostic value of MGMT promoter status by pyrosequencing assay for glioblastoma patients' survival: a meta-analysis. World J Surg Oncol. 2016;14:261.

18. Jiang T, Mao Y, Ma W, et al. CGCG clinical practice guidelines for the management of adult diffuse gliomas. Cancer Lett. 2016;375:263-73.

19. Malley DS, Hamoudi RA, Kocialkowski S, et al. A distinct region of the MGMT CpG island critical for transcriptional regulation is preferentially methylated in glioblastoma cells and xenografts. Acta Neuropathol. 2011;121:651-61.

20. Mur P, Rodriguez de Lope A, Diaz-Crespo FJ, et al. Impact on prognosis of the regional distribution of MGMT methylation with respect to the $\mathrm{CpG}$ island methylator phenotype and age in glioma patients. J Neurooncol. 2015;122:441-50.

21. Dunn J, Baborie A, Alam F, et al. Extent of MGMT promoter methylation correlates with outcome in glioblastomas given temozolomide and radiotherapy. Br J Cancer. 2009;101:124-31.

22. Brigliadori G, Foca F, Dall'Agata M, et al. Defining the cutoff value of MGMT gene promoter methylation and its predictive capacity in glioblastoma. J Neurooncol. 2016;128:333-9.

23. Weller M, Stupp R, Reifenberger G, et al. MGMT promoter methylation in malignant gliomas: ready for personalized medicine? Nat Rev Neurol. 2010;6:39-51.

24. Zhang W, Zhang J, Hoadley K, et al. miR-181d: a predictive glioblastoma biomarker that downregulates MGMT expression. Neuro Oncol. 2012;14:712-9.

25. Cheng W, Ren X, Zhang C, et al. Bioinformatic profiling identifies an immune-related risk signature for glioblastoma. Neurology. 2016;86:2226-34.

26. Chai R, Zhang K, Wang K, et al. A novel gene signature based on five glioblastoma stem-like cell relevant genes predicts the survival of primary glioblastoma. J Cancer Res Clin Oncol. 2018;144:439-47.

27. Wick W, Meisner C, Hentschel B, et al. Prognostic or predictive value of MGMT promoter methylation in gliomas depends on IDH1 mutation. Neurology. 2013;81:1515-22.

28. Mulholland S, Pearson DM, Hamoudi RA, et al. MGMT CpG island is invariably methylated in adult astrocytic and oligodendroglial tumors with IDH1 or IDH2 mutations. Int J Cancer. 2012;131:1104-13.

29. Louis DN, Ohgaki H, Wiestler OD, Cavenee WK. WHO Classification of Tumours of the Central Nervous System. WHO Classification of Tumours, Revised 4th edition. Volume 1. France: International Agency for Research on Cancer; 2016.
30. Reifenberger G, Hentschel B, Felsberg J, et al. Predictive impact of MGMT promoter methylation in glioblastoma of the elderly. Int J Cancer. 2012;131:1342-50.

31. Wiewrodt D, Nagel G, Dreimuller N, et al. MGMT in primary and recurrent human glioblastomas after radiation and chemotherapy and comparison with p53 status and clinical outcome. Int J Cancer. 2008;122:1391-9.

32. Herman JG, Graff JR, Myohanen S, Nelkin BD, Baylin SB. Methylation-specific PCR: a novel PCR assay for methylation status of CpG islands. Proc Natl Acad Sci USA. 1996;93: 9821-6.

33. Vlassenbroeck I, Califice S, Diserens AC, et al. Validation of realtime methylation-specific PCR to determine O6-methylguanineDNA methyltransferase gene promoter methylation in glioma. $\mathrm{J}$ Mol Diagn. 2008;10:332-7.

34. Wojdacz TK, Dobrovic A. Methylation-sensitive high resolution melting (MS-HRM): a new approach for sensitive and highthroughput assessment of methylation. Nucleic Acids Res. 2007;35:e41.

35. Jeuken JW, Cornelissen SJ, Vriezen M, et al. MS-MLPA: an attractive alternative laboratory assay for robust, reliable, and semiquantitative detection of MGMT promoter hypermethylation in gliomas. Lab Invest. 2007;87:1055-65.

36. Bady P, Sciuscio D, Diserens AC, et al. MGMT methylation analysis of glioblastoma on the Infinium methylation BeadChip identifies two distinct $\mathrm{CpG}$ regions associated with gene silencing and outcome, yielding a prediction model for comparisons across datasets, tumor grades, and CIMP-status. Acta Neuropathol. 2012;124:547-60.

37. Bady P, Delorenzi M, Hegi ME. Sensitivity analysis of the MGMT-STP27 model and impact of genetic and epigenetic context to predict the MGMT methylation status in gliomas and other tumors. J Mol Diagn. 2016;18:350-61.

38. Wemmert S, Ketter R, Rahnenfuhrer J, et al. Patients with highgrade gliomas harboring deletions of chromosomes $9 p$ and $10 q$ benefit from temozolomide treatment. Neoplasia. 2005;7:883-93.

39. Herrlinger U, Rieger J, Koch D, et al. Phase II trial of lomustine plus temozolomide chemotherapy in addition to radiotherapy in newly diagnosed glioblastoma: UKT-03. J Clin Oncol. 2006;24:4412-7.

40. Xia D, Reardon DA, Bruce JL, Lindeman NI. The clinical implications of inconsistently methylated results from glioblastoma MGMT testing by replicate methylation-specific PCR. J Mol Diagn. 2016;18:864-71.

41. Christians A, Hartmann C, Benner A, et al. Prognostic value of three different methods of MGMT promoter methylation analysis in a prospective trial on newly diagnosed glioblastoma. PLoS One. 2012;7:e33449.

42. Chai RC, Zhang KN, Liu YQ, et al. Combinations of four or more $\mathrm{CpGs}$ methylation present equivalent predictive value for MGMT expression and temozolomide therapeutic prognosis in gliomas. CNS Neurosci Ther. 2018. https://doi.org/10.1111/cns. 13040.

43. Ramalho-Carvalho J, Pires M, Lisboa S, et al. Altered expression of MGMT in high-grade gliomas results from the combined effect of epigenetic and genetic aberrations. PLoS One. 2013;8:e58206.

44. Felsberg J, Rapp M, Loeser S, et al. Prognostic significance of molecular markers and extent of resection in primary glioblastoma patients. Clin Cancer Res. 2009;15:6683-93. 